\title{
Diagnóstico situacional como ferramenta para o fortalecimento da
}

\section{interprofissionalidade}

\author{
Situational diagnosis as a tool for strengthening interprofessionality \\ El diagnóstico situacional como herramienta para fortalecer la interprofesionalidad
}

Lorrane Nere de Lima

ORCID: https://orcid.org/0000-0002-8930-294X Universidade Federal de Sergipe, Brasil

E-mail: lorranenere@ hotmail.com

Iara Santana Targino

ORCID: https://orcid.org/0000-0002-0986-4513 Universidade Federal de Sergipe, Brasil

E-mail: iarasantanatg@gmail.com

Ana Letícia Soares dos Reis Santos

ORCID: https://orcid.org/0000-0003-1044-0205 Universidade Federal de Sergipe, Brasil E-mail: anareis.fs@gmail.com

Isabella Meneses de Queiroz Marques

ORCID: https://orcid.org/0000-0002-7571-5719 Universidade Federal de Sergipe, Brasil E-mail: isabellameneses13@gmail.com

Ana Silvia Moccellin

ORCID: https://orcid.org/0000-0002-9195-4226 Universidade Federal de Sergipe, Brasil E-mail: ana.moccellin@academico.ufs.br

Maria do Carmo de Oliveira Ribeiro

ORCID: https://orcid.org/0000-0003-4719-3893 Universidade Federal de Sergipe, Brasil E-mail: enffer2@gmail.com

\begin{abstract}
Resumo
O Diagnóstico Situacional é considerado uma ferramenta potencial para o fortalecimento da Interprofissionalidade. Dessa forma, o objetivo do presente estudo foi descrever a importância desta ferramenta para o fortalecimento da interprofissionalidade entre os estudantes com o intuito de despertar o interesse da Estratégia Saúde da Família quanto a temática, associado ao anseio de uma prática humanizada preconizada pelas diretrizes do Sistema Único de Saúde. Os instrumentos utilizados na coleta de dados foram a Readiness for Interprofessional Learning Scale (RIPLS), escala que avalia a adesão da interprofissionalidade pelos estudantes e um questionário complementar da situação socioeconômica e demográfica, ambos aplicados entre julho e agosto de 2020 através da plataforma Google Forms e com assinatura online do termo de consentimento ( $\mathrm{n}^{\circ}$ do parecer 3.623.996). Os resultados da RIPLS foram positivos à interprofissionalidade nos três fatores avaliados: trabalho em equipe e colaboração (fator 1), identidade profissional (fator 2) e atenção à saúde centrada no paciente (fator 3). $\mathrm{O}$ fator 2 obteve a menor pontuação. Concluiu-se que o diagnóstico situacional é uma ferramenta capaz de fortalecer a interprofissionalidade ao possibilitar que estudantes, professores e profissionais de saúde compactuem suas experiências e conhecimentos para solucionar os problemas de saúde advindos da comunidade.
\end{abstract}

Palavras-chave: Atenção primária à saúde; Avaliação em saúde; Educação interprofissional; Estratégia saúde da família; Estudos de avaliação como assunto.

\begin{abstract}
The situational diagnosis is considered a potential tool for strengthening interprofessionality. Therefore, the objective of the present study was to describe its importance to strengthen interprofessionality among students in order to increase the interest of the Family Health Strategy in the theme in association with the claim for a humanized practice, as recommended by the guidelines of the Unified Health System. The instruments used in data collection were the Readiness for Interprofessional Learning Scale (RIPLS), a scale that assesses students' adherence to interprofessionality and a complementary questionnaire on the socioeconomic and demographic situation, both applied between July and August of 2020 through the Google Forms platform with online signature of the consent form (number 3.623.996). The results of RIPLS were positive for interprofessionality in the three factors evaluated: teamwork and collaboration (factor 1), professional identity (factor 2) and patient-centered health care (factor 3 ). The
\end{abstract}


factor 2 got the lowest score. It was concluded that situational diagnosis is a tool capable of strengthening interprofessionality by enabling students, teachers and health professionals to combine their experiences and knowledge to solve health problems arising from the community.

Keywords: Primary health care; Health evaluation; Interprofessional education; Family health strategy; Evaluation studies as topic.

\section{Resumen}

El Diagnóstico Situacional se considera una herramienta potencial para fortalecer la Interprofesionalidad. Así, el objetivo de este estudio fue describir la importancia de esta herramienta para el fortalecimiento de la interprofesionalidad entre los estudiantes con el fin de despertar el interés de la Estrategia de Salud de la Familia sobre el tema, asociado al deseo de una práctica humanizada recomendada por los lineamientos del Sistema. Unidad de Salud. Los instrumentos utilizados en la recolección de datos fueron la Readiness for Interprofessional Learning Scale (RIPLS), escala que evalúa la adherencia de los estudiantes a la interprofesionalidad y un cuestionario complementario sobre la situación socioeconómica y demográfica, ambos aplicados entre julio y agosto de 2020 a través de la plataforma Google Forms y con firma online del formulario de consentimiento (dictamen $\mathrm{n}^{\circ}$ 3.623.996). Los resultados del RIPLS fueron positivos para la interprofesionalidad en los tres factores evaluados: trabajo en equipo y colaboración (factor 1), identidad profesional (factor 2) y atención sanitaria centrada en el paciente (factor 3). El factor 2 tuvo la puntuación más baja. Se concluyó que el diagnóstico situacional es una herramienta capaz de fortalecer la interprofesionalidad al permitir que estudiantes, docentes y profesionales de la salud compartan sus experiencias y conocimientos para resolver los problemas de salud que se presentan en la comunidad.

Palabras clave: Atención primaria de salud; Evaluación de la salud; Educación interprofesional; Estrategia de salud de la família; Estudios de evaluación como tema.

\section{Introdução}

A Atenção Primária à Saúde (APS) é um componente da Rede de Atenção à Saúde (RAS) que se encontra em maior alcance da população, sendo considerada a porta de entrada para os serviços de assistência. A APS tem como propósito assegurar os princípios que fundamentam o Sistema Único de Saúde (SUS), garantindo a todos os brasileiros a criação de vínculo com as unidades de saúde, corresponsabilização dos atores e o acesso a saúde integral e contínua (Ministério da Saúde, 2012).

O aumento da oferta de ações e serviços para a população que ocorreu nos últimos 20 anos se deu através da expansão e da manutenção da cobertura das Equipes de Saúde da Família (eSF) (Facchini, Tomasi \& Dilélio, 2018). Para isso uma das ferramentas utilizadas no contexto da atuação das eSF configura-se no Diagnóstico Situacional (DS), instrumento imprescindível no levantamento das principais necessidades de saúde da comunidade e processos de trabalho a fim de subsidiar a programação e planejamento das ações (Ribeiro, Pedrosa, Nogueira \& Souza, 2012).

Neste sentido, a busca pela integralidade tem impulsionado as discussões acerca da formação dos profissionais da área da saúde que seguia, predominantemente, o modelo uniprofissional, ou seja, baseado no aprendizado específico da profissão, havendo apenas o contato entre estudantes do próprio curso. Esse modelo é tido como um obstáculo para alcançar os objetivos da APS sendo um entrave à reorientação da assistência à saúde, uma vez que se pretende superar o modelo biomédico pautado na concepção biológica da doença, fragmentação do cuidado e em práticas curativas (Peduzzi, Norman, Germani, Silva \& Souza, 2013). Percebendo esta demanda, a temática sobre a preparação de recursos humanos para o trabalho em saúde ganhou espaço nas discussões de Conferências Nacionais de Saúde propondo mudanças curriculares nos cursos da área (Dias, 2013).

A EIP acontece quando dois ou mais estudantes de diferentes cursos, ao se relacionarem em grupo, aprendem conjuntamente sobre as competências e saberes de cada profissão, abrindo espaço para valorização e respeito mútuo, diferente da educação multiprofissional no qual não há precisamente esta interação. Desta forma, a EIP possibilita o desenvolvimento de habilidades para o trabalho em equipe e a participação colaborativa resultem em uma melhor qualidade na assistência (Reeves, Perrier, Goldman, Freeth \& Zwarenstein, 2013; Peduzzi et al., 2013).

No Brasil, uma das iniciativas para a implementação da EIP ocorreu por meio do Programa de Educação pelo Trabalho para a Saúde (PET-Saúde), que, objetiva "favorecer a indissociabilidade entre ensino, pesquisa e extensão, a 
constituição de grupos de aprendizagem tutorial, a interdisciplinaridade e a integração ensino-serviço, incluindo um plano de pesquisa em atenção básica" (Dias, 2013).

Diante disso, o PET-Saúde possibilita aos estudantes da Universidade Federal de Sergipe (UFS) o acompanhamento e vivência das práticas profissionais, do trabalho em equipe e participação em atividades educativas nas UBSs, nos Centros de atenção psicossocial (CAPS) e na gestão da Secretaria Municipal de Saúde do município de São Cristóvão, tudo isso em prol de melhorias na assistência, permitindo que estudantes de diferentes cursos desenvolvam suas habilidades em conjunto, tema da edição do programa em 2018/2019, PET-Saúde Interprofissionalidade (Ministério da Saúde, 2018).

Nesta perspectiva, durante o primeiro ano do PET-Saúde, os grupos tutoriais puderam realizar o DS do território de abrangência das UBSs e (CAPS), além da gestão da Secretaria Municipal de Saúde do município de São Cristóvão.

Após buscas nas bases de dados indexadas Scielo (Scientific Eletronic Library Online), Biblioteca Virtual em Saúde (BVS) e PubMed, identificou-se carência de estudos sobre a temática. A partir de então, estabeleceu-se a seguinte questão norteadora: o diagnóstico situacional, sendo uma ferramenta que necessita do trabalho articulado e conjunto de diferentes atores das equipes contribui com a consolidação do trabalho interprofissional?

Dessa maneira, o objetivo dessa pesquisa é descrever a importância do diagnóstico situacional como ferramenta para o fortalecimento da interprofissionalidade entre os estudantes com o intuito de despertar o interesse da eSF quanto a temática, associado ao anseio de uma prática humanizada, conforme preconizada pelas diretrizes do SUS.

Espera-se que os resultados advindos desse estudo possam auxiliar a elaboração de um projeto aplicativo a ser implementado nas áreas de atuação do PET-Saúde e que ofereçam novos rumos para a prática da interprofissionalidade.

\section{Metodologia}

Trata-se de um estudo descritivo com abordagem quantitativa. Esse tipo de pesquisa descreve uma realidade por meio de dados numéricos a fim de avaliar determinados comportamentos de indivíduos ou grupos (Lakatos \& Marconi, 2010).

A amostra foi do tipo consecutiva, probabilística e por conveniência. A casuística foi composta por todos que aceitaram o Termo de Consentimento Livre e Esclarecido (TCLE) presente no formulário eletrônico construído no Google Forms®. Participaram do estudo 34 estudantes da UFS dos cursos de educação física, enfermagem, farmácia, fisioterapia, fonoaudiologia, medicina, nutrição, odontologia e serviço social que fazem parte do PET-Saúde Interprofissionalidade. A amostra final foi constituída por 31 estudantes, pois três estudantes que fazem parte do projeto e que participaram da construção deste estudo foram excluídas da amostra.

A coleta de dados ocorreu durante os meses de julho e agosto de 2020. O formulário foi construído na plataforma online Google Forms ${ }^{\circledR}$ e dividido em duas partes. A primeira parte foi destinada à caracterização socioeconômica dos estudantes através de perguntas abertas e de múltipla escolha acerca do gênero, raça/cor, situação conjugal, situação de moradia, município de residência, idade, curso de graduação e sobre a importância da bolsa paga pelo projeto na manutenção acadêmica dos estudantes. Na segunda, o participante deveria responder a Readiness for Interprofessional Learning Scale (RIPLS), instrumento criado por Parsell e Bligh (1999), traduzido para a língua portuguesa e validado por Peduzzi, Norman, Coster e Meireles (2015) para a avaliação da EIP.

A RIPLS consiste em 27 itens divididos entre três fatores: (1) trabalho em equipe e colaboração, refletida em 14 itens (itens 1-9; 12-16) acerca da disponibilidade para trabalho em equipe e aprendizado com outros profissionais da saúde; (2) Identidade profissional, com oito itens no total (itens 10;11;18-22), dentre as quais, cinco (itens 10;11;17;19; 21 ) fazem alusão à posicionamentos negativos em relação a EIP; e a última área, (3) atenção à saúde centrada no paciente, com 5 itens (itens 23-27) que refletem a disponibilidade em compreender as necessidades dos pacientes (Peduzzi et al., 2015). 
As respostas para a RIPLS foram configuradas de acordo com o formato da escala Likert de cinco pontos, cuja pontuação varia entre discordo totalmente (1) e concordo totalmente (5). A análise foi feita a partir do cálculo das pontuações individuais por fator e global. A pontuação global pode variar entre 27 e 135, e as pontuações máximas para os fatores 1,2 e 3 são, respectivamente, 70, 40 e 25; considerando que quanto maior for a pontuação, maior a disponibilidade e quanto menor a pontuação, menor a disponibilidade dos estudantes para a EIP (Barbosa, 2020).

Os dados foram analisados e organizados em planilha do Microsoft Excel® 2013. Foram calculadas as médias da pontuação global e da pontuação por fator. Para avaliar a adesão dos estudantes à EIP observou-se a adequação da pontuação média obtida na escala em relação à pontuação máxima possível para cada fator, sendo 70, 40 e 25 equivalentes a 100\% de adesão à EIP nos fatores 1, 2 e 3, respectivamente, e 135 na pontuação global.

Os itens da RIPLS que exemplificam atitudes negativas na EIP são: 10 "Não quero desperdiçar meu tempo aprendendo junto com estudantes de outras profissões da saúde. “, 11 "Não é necessário que estudantes de graduação da área da saúde aprendam juntos.”, 17 “A função dos demais profissionais da saúde é principalmente apoio aos médicos?”, 19 "Eu me sentiria desconfortável se outro estudante da área da saúde soubesse mais sobre um tópico do que eu." e 21 "Chegar a um diagnóstico será a principal função do meu papel profissional (objetivo clínico)”. Logo, os escores desses itens descritos tiveram pontuação invertida para a análise dos dados.

Em todas as etapas desta pesquisa foi seguida a Resolução 466/2012 do Conselho Nacional de Saúde (CNS). Trata-se de um projeto guarda-chuva intitulado "Estratégias de intervenção a partir de determinantes das condições de vida e estado de saúde de famílias adscritas às USF dos municípios de Aracaju/SE e São Cristóvão/SE”, previamente aprovado pelo Comitê de Ética e Pesquisa da Universidade Federal de Sergipe sob parecer nº 3.623.996 (CAAE 76850817.9.0000.5546).

\section{Resultados}

A amostra foi constituída por 34 estudantes que atenderam aos critérios de elegibilidade, sendo que três alunas foram excluídas por serem participantes do estudo, resultando uma amostra de 31 discentes. Na Tabela 1 é demonstrado a quantidade de participantes a partir das suas características sociodemográficas. 
Tabela 1. Características sociodemográficas dos estudantes da Universidade Federal de Sergipe participantes do PET-Saúde Interprofissionalidade, 2020.

\begin{tabular}{|c|c|c|}
\hline & $n$ & $\%$ \\
\hline TOTAL & 31 & 100 \\
\hline \multicolumn{3}{|l|}{ Gênero } \\
\hline Feminino & 23 & 74,2 \\
\hline Masculino & 8 & 25,8 \\
\hline \multicolumn{3}{|l|}{ Raça/cor Autodeclarada } \\
\hline Parda & 21 & 67,7 \\
\hline Preta & 5 & 16,1 \\
\hline Branca & 5 & 16,1 \\
\hline \multicolumn{3}{|l|}{ Situação conjugal } \\
\hline Solteiro & 29 & 93,5 \\
\hline União Estável & 2 & 6,5 \\
\hline \multicolumn{3}{|l|}{ Município de moradia } \\
\hline Aracaju & 17 & 54,8 \\
\hline São Cristóvão & 9 & 29 \\
\hline Nossa Senhora do Socorro & 2 & 6,5 \\
\hline Barra dos Coqueiros & 1 & 3,2 \\
\hline Outros & 2 & 6,5 \\
\hline \multicolumn{3}{|l|}{ Curso } \\
\hline Educação física & 3 & 9,7 \\
\hline Enfermagem & 5 & 16,1 \\
\hline Farmácia & 4 & 12,9 \\
\hline Fisioterapia & 5 & 16,1 \\
\hline Fonoaudiologia & 2 & 6,5 \\
\hline Medicina & 3 & 9,7 \\
\hline Nutrição & 2 & 6,5 \\
\hline Odontologia & 4 & 12,9 \\
\hline Serviço Social & 3 & 9,7 \\
\hline \multicolumn{3}{|l|}{ Período } \\
\hline $6^{\circ}$ período & 10 & 32,3 \\
\hline $7^{\circ}$ período & 6 & 19,4 \\
\hline $8^{\circ}$ período & 5 & 16,1 \\
\hline $5^{\circ}$ período & 5 & 16,1 \\
\hline $4^{\circ}$ período & 3 & 9,7 \\
\hline $9^{\circ}$ período & 2 & 6,5 \\
\hline \multicolumn{3}{|l|}{ Situação de Moradia } \\
\hline Moro sozinho & 3 & 9,7 \\
\hline Divido moradia & 7 & 22,6 \\
\hline Moro com pais/familiares & 20 & 64,5 \\
\hline Moradia universitária & 1 & 3,2 \\
\hline
\end{tabular}

Fonte: São Cristóvão (2020).

São auto identificados como pardas (os) (67,7\%), solteiros (93,5\%), residentes do município de Aracaju-SE (54,8\%), cursam o sexto período da graduação $(32,3 \%)$ e moram com os pais e/ou familiares $(64,5 \%)$. Conforme dados da Tabela 2 , a amostra é composta por 29 estudantes bolsistas $(93,5 \%)$ e 2 estudantes voluntários $(6,5 \%)$. 
Tabela 2. Impacto financeiro da bolsa PET-Saúde Interprofissionalidade na manutenção dos estudantes da UFS, 2020.

\begin{tabular}{|c|c|c|}
\hline & $n$ & $\%$ \\
\hline TOTAL & 31 & 100 \\
\hline \multicolumn{3}{|c|}{ "Você é aluno bolsista ou voluntário do PET-Saúde Interprofissionalidade?" } \\
\hline Bolsista & 29 & 93,5 \\
\hline Voluntário & 2 & 6,5 \\
\hline \multicolumn{3}{|c|}{ "A sua participação no projeto depende do pagamento da bolsa?” } \\
\hline Sim & 15 & 48,4 \\
\hline Não & 14 & 45,2 \\
\hline Não sou bolsista & 2 & 6,5 \\
\hline \multicolumn{3}{|c|}{$\begin{array}{l}\text { "Você destina o valor da bolsa para o pagamento de contas essenciais como } \\
\text { aluguel, alimentação, transporte, medicação, água ou energia?" }\end{array}$} \\
\hline Sim & 22 & 71 \\
\hline Não & 7 & 22,6 \\
\hline Não sou bolsista & 2 & 6,5 \\
\hline \multicolumn{3}{|c|}{ "A bolsa é essencial para a sua permanência na universidade?" } \\
\hline Sim & 18 & 58,1 \\
\hline Não & 11 & 35,5 \\
\hline Não sou bolsista & 2 & 6,5 \\
\hline
\end{tabular}

Fonte: São Cristóvão (2020).

Observou-se que $48,4 \%$ dos estudantes afirmam que a sua participação no projeto é dependente do pagamento da bolsa e $71 \%$ afirmam que destinam essa quantia para o pagamento de contas essenciais como aluguel, alimentação, transporte, água ou energia. Ainda, 58,1\% dos estudantes afirmam que a bolsa paga pelo projeto é essencial para a sua permanência na universidade (Tabela 2). A Tabela 3 traz os resultados relativos à aplicação da RIPLS.

Tabela 3. Avaliação da adesão à Educação Interprofissional pelos estudantes da UFS participantes do PET-Saúde Interprofissionalidade, 2020.

\begin{tabular}{lcccc}
\hline & Pontuação máxima da RIPLS & \multicolumn{2}{c}{ Adesão à EIP } \\
\hline & $\mathrm{PF}^{*}$ & $\%$ & Média & $\%$ \\
\hline Pontuação global & 135 & 100 & 118,4 & 87,7 \\
Fator 1 & 70 & 100 & 63,9 & 91,3 \\
Fator 2 & 40 & 100 & 31,3 & 78,2 \\
Fator 3 & 25 & 100 & 23,2 & 92,6 \\
\hline
\end{tabular}

*PF: Pontuação máxima por fator. Fonte: São Cristóvão (2020).

A pontuação média apresentada para o fator 1, que reflete as dimensões da EIP no trabalho em equipe e colaboração, apresentou 91,3\% de adesão à pontuação máxima para este quesito. O fator 2 apresentou 78,2\% de adesão à EIP nos termos que fazem relação com a identidade profissional. Já o fator 3, que reflete a atenção à saúde centrada no paciente, apresentou $92,6 \%$ de adesão à pontuação máxima (Tabela 3). A pontuação global, que pode variar entre 27 e 135 , apresentou valor médio de 118,4 neste estudo, o que representa uma adesão de $87,7 \%$ ao valor máximo.

\section{Discussão}

A formação para o trabalho em saúde tem sido amplamente discutida em nível global pelas organizações responsáveis para que ocorra a readequação dos processos assistenciais às mudanças no modelo de saúde, na pretensão de superar os problemas que requerem cuidados contínuos, integrais e que considerem a complexidade individual e coletiva dos sujeitos (Sagahutu, Kagwiza, Cilliers \& Jelsma, 2020). Desta forma, instituições de ensino têm possibilitado a imersão de estudantes de diferentes cursos da área da saúde em contextos assistenciais que requer o trabalho em equipe, para introduzir a abordagem da EIP em suas formações (Lima et al., 2020). Logo, este estudo retrata a vivência de estudantes em um programa de EIP através 
da perspectiva da construção do DS que se mostrou eficaz no fortalecimento da Interprofissionalidade.

No Brasil, a criação da Secretaria de Gestão do Trabalho e da Educação na Saúde (SGTES), em 2003, foi um marco para a efetivação da EIP. O setor instituído no âmbito do Ministério da Saúde torna factível a integração entre ensino-serviço, até então possível através da parceria com Ministério da Educação, possibilitando que programas como o PET-saúde consolidem as estratégias de EIP (Peduzzi et al., 2013; Dias, 2013).

Além disso, visto que o PET-Saúde é financiado pelo Ministério da Saúde, os resultados demonstrados certificam a importância desse subsídio, posto que metade dos alunos condicionam sua participação no projeto à assistência financeira recebida e 58,1\% à sua permanência na Universidade. Tal recurso é importante para superar os obstáculos que impedem um bom desempenho acadêmico, diminuindo assim o percentual de abandono e trancamento do curso, pois diversos acadêmicos necessitam de recursos mínimos para a sobrevivência estudantil como moradia, alimentação, transporte e recursos financeiros (Vasconcelos, 2010).

O PET-Saúde Interprofissionalidade desenvolvido na RAS de São Cristóvão - Sergipe conjuntamente com a UFS, iniciou-se em abril de 2019. Os estudantes petianos são dos cursos de educação física, enfermagem, farmácia, fisioterapia, fonoaudiologia, medicina, nutrição, odontologia e serviço social, que estiveram sob a preceptoria dos profissionais da saúde do município que atuam na RAS e sob orientação dos professores da área da saúde da UFS. As atividades do PET-Saúde são desenvolvidas semanalmente conforme cronograma desenvolvido pela coordenação geral do programa.

Portanto, os estudantes foram inseridos na RAS e instruídos a construírem o DS das UBS, CAPS e Secretaria de Saúde a qual foram alocados, cada grupo tutorial multiprofissional realizou a cobertura dos seus territórios de abrangência visando estimular o diálogo entre os participantes das diferentes áreas da saúde, e consequentemente, a interprofissionalidade.

O DS constitui uma ferramenta de análise das condições de saúde e adoecimento individuais e coletivas da população de uma determinada região, levando-se em consideração a complexidade do processo de saúde-doença sustentada pelos determinantes sociais de saúde, também objetiva identificar problemas, limitações e potencialidades dos serviços de saúde para planejar ações de adequação do sistema às necessidades da população (Silva, Koopmans \& Daher, 2016; Queiroz \& Valente, 2019). A realização do DS se detém da coleta de dados e reconhecimento do território, que além de contar com a participação dos profissionais de saúde devem ser obtidos através da população (Ribeiro et al., 2012; Silva et al., 2016). Para tanto, a elaboração do DS teve como propósito descrever e identificar os problemas de saúde da população para definir uma intervenção que pudesse trazer melhorias para as unidades de saúde.

Os programas de EIP têm se mostrado favoráveis para o desenvolvimento de competências colaborativas e de comunicação entre estudantes de diferentes cursos da área de saúde (Wang, Feng, Gao \& Yang, 2019), no entanto, ainda há poucas evidências que correlacionem a efetividade desses programas a qualidade na prestação de serviços nos sistemas de saúde (Kanji, Lin \& Karan, 2020). Considerando os benefícios da EIP, a Organização Mundial de Saúde (OMS) apoia a iniciativa e a reconhece como uma etapa essencial na preparação dos estudantes para uma prática profissional adequada as necessidades da população e demandas de saúde locais. Além de possibilitar que estudantes tenham experiências de vivência na realidade do sistema de saúde e comunidade e aprendam uns com os outros, compartilhando conhecimentos sobre seus cursos para tornarem-se profissionais aptos à prática colaborativa (World Health Organization, 2010).

Nesse contexto, no qual se pretende estimular a interprofissionalidade é possível medir as atitudes dos estudantes referentes a exposição a essas experiências de EIP. Um instrumento muito utilizado nessa perspectiva é a RIPLS, escala validade que objetiva avaliar a prontidão para o trabalho interprofissional, ou seja, é capaz de identificar a disposição dos estudantes para o aprendizado compartilhado com outros estudantes de diferentes cursos (Kanji et al., 2020).

As competências avaliadas da RIPLS estão divididas em três fatores (Peduzzi et al., 2015). O fator 1, trabalho em equipe e colaboração, os estudantes obtiveram uma prontidão positiva, sendo o segundo fator com maior pontuação. 
Subentende-se diante desse resultado que os estudantes entendem a importância do aprendizado compartilhado, de estabelecer uma boa comunicação com profissionais de saúde de outras áreas e a importância do trabalho em equipe na resolução de problemas e de dispor de boas relações interpessoais. Resultados semelhantes foi descrito em outro estudo, numa revisão sistemática de sete artigos que avaliou as atividades de EIP durante a graduação, com alunos de três ou mais cursos da saúde (Dyess, Brown, Brown, Flautt \& Barnes, 2019).

O fator 2, que avalia a identidade profissional, teve a menor pontuação entre os fatores, apesar de estar acima da média do que é considerado desejável, podendo-se inferir que ainda existe uma certa resistência no aprendizado compartilhado apontando para uma possível atitude de competição e autonomia profissional e um direcionamento para um objetivo majoritariamente clínico, que podem constituir barreiras para EIP. Segundo uma pesquisa que avaliou uma experiência de EIP utilizando a RIPLS com alunos dos cursos de medicina, assistente médico e enfermagem, antes e depois de uma viagem para América Central (em um local carente de assistência médica), foi percebido uma queda nas atitudes nos fatores um e dois. Diante desses resultados conclui-se que a mudança de atitude antes e depois do evento poderia estar relacionado a uma idealização prévia do evento e a inserção precoce dessa experiência nos cursos mencionados, quando os estudantes ainda não têm os conhecimentos suficientes para assumir seus papéis e atuar coordenadamente com o grupo (Skolka, Hennrikus, Khalid \& Hennrikus, 2020).

Ademais, ainda não é consenso em que ano os estudantes devem passar por uma experiência de EIP, pois existe algumas vertentes que defendem uma inserção precoce e outras mais tardiamente (Lima et al., 2020). Porém, percebe-se que quando exposta desde os períodos iniciais a EIP pode possibilitar uma maior aceitação desta modalidade de ensino. O edital PET-Saúde Interprofissionalidade contemplou graduandos dos períodos iniciais de seus cursos, apesar da menor pontuação no fator 2, acredita-se que isso ajuda os alunos a identificarem suas limitações e as dificuldade do trabalho em equipe e auxilie na adaptação dessa nova forma de aprendizagem, multidisciplinar e interprofissional. Em suma, a EIP quando apresentada no início da graduação favorece o respeito entre as profissões e a quebra de estereótipos arraigados, no entanto ela pode ser mais eficaz se apresentada de forma contínua e gradativa durante a graduação (Kanji et al., 2020; Skolka et al., 2020).

Por fim, o fator três que expressa sobre a atenção à saúde centrada no paciente, atingiu a maior pontuação entre os fatores, demonstrando a sensibilização dos estudantes para os cuidados em saúde mais humanizados. Diante disso, acredita-se que o trabalho interprofissional é movido por um objetivo comum, o cuidado voltado ao paciente, que supera os desafios decorrentes do trabalho em equipe (Skolka et al., 2020). No SUS esse conceito é ainda mais marcante, pois as atribuições dos profissionais de saúde vão além das fronteiras do consultório, devendo as equipes assumir a responsabilidade pelas famílias presentes em seus territórios de abrangência.

Nessa perspectiva as ações são voltadas a comunidade de tal forma que a equipe deve levar informação e saúde até a mesma, seja com ações de caráter preventivo como também assistências que podem ser realizadas nas instituições de saúde, no domicílio das famílias, nas escolas, nas campanhas que ocorrem na rua ou em salões comunitários (Ministério da Saúde, 2012), como foi vivenciado pelos estudantes durante o primeiro ano do PET-Saúde interprofissionalidade ao acompanhar e auxiliar as ações de saúde das equipes. Logo, o DS auxilia também na efetivação da reorientação da atenção à saúde instituído com a implementação da ESF nas UBSs, possibilitando que as equipes multiprofissionais tracem o planejamento estratégico em saúde que priorizem as maiores necessidades da comunidade (Ribeiro, Ribeiro, Dias, Matos \& Ferreira, 2008).

$\mathrm{Na}$ prática, o trabalho interprofissional pode também gerar conflitos, muitos desses, atrelados a falta de esclarecimento dos papéis e responsabilidades por parte dos membros da equipe, a comunicação mal estabelecida, escassez das relações interpessoais, isolamento em grupos, hierarquia entre os profissionais da saúde e preconceitos em relação ao próprio curso e a dos demais (Skolka et al., 2020; Nguyen, Smith, Hunter \& Harnett, 2019; Furr, 2020). Outro ponto importante, que limita a efetivação da EIP, são os distintos horários dos currículos dos cursos da saúde, inviabilizando um horário comum para 
realização das atividades que requer a participação de estudantes de diferentes cursos (Correa, Hermuche, Luccheti, Ezequiel \& Luccheti, 2019).

Embora haja uma crescente incorporação de programas de EIP no Brasil, muitos ainda não são de caráter obrigatório dificultando a apresentação dessa modalidade de ensino aos estudantes. Somado a isso, destaca-se também outras barreiras neste processo de implementação da EIP, tais como o currículo heterogêneo, principalmente em relação ao curso de medicina, que em parte poderia justificar a menor disposição desses alunos para as práticas interprofissionais; baixa integração e comunicação entre os cursos; baixo incentivo financeiro e poucas iniciativas pelo corpo acadêmico (Correa et al., 2019).

A necessidade de implementação de atividades de EIP nos cursos da área da saúde está bem estabelecida, sendo uma iniciativa reconhecida e apoiado pelas organizações de saúde (Kanji et al., 2020; World Health Organization, 2010; Dyess et al., 2019). No Brasil, percebe-se a necessidade da incorporação da EIP nos currículos dos cursos da área, com metodologias ativas de ensino e a inserção dos discentes na RAS em especial na APS, onde os alunos podem desenvolver atividades práticas na comunidade (Correa et al., 2019).

Para tanto, são necessários mais estudos com diferentes metodologias que possam captar as percepções e mudanças de atitudes dos estudantes, além de superar as limitações metodológicas deste estudo, decorrentes do pequeno tamanho amostral e da ausência de avaliação prévia das atividades de EIP, dentre outros critérios que devem assegurar a qualidade dos resultados e, desta forma, investigar com precisão o impacto do programa.

\section{Conclusão}

Conclui-se que o DS é uma ferramenta capaz de fortalecer a interprofissionalidade ao possibilitar que estudantes, professores e profissionais de saúde compactuem suas experiências e conhecimentos para solucionar os problemas de saúde advindos da comunidade após imersão na mesma, e desse modo ampliar a visão da saúde em todos seus ramos, representados pelos diferentes cursos da área da saúde, para desta forma convergirem seus conhecimentos em busca da integralidade do cuidado. Com isso, os estudantes ainda no processo de formação podem desenvolver competências e habilidades para o trabalho em equipe e colaboração, além de possibilitar uma visão ampliada dos problemas de saúde da população e dificuldades dos serviços de saúde. Sugerem-se novos estudos com outras abordagens metodológicas e grupos populacionais distintos que possam identificar a importância do DS para o fortalecimento da interprofissionalidade.

\section{Agradecimentos}

Agradecemos ao Ministério da Saúde pela oportunidade de vivenciarmos o PET-Saúde Interprofissionalidade e à Prefeitura do município de São Cristóvão, em especial à Secretaria Municipal de Saúde, por ter sido a sede de desenvolvimento desse trabalho.

\section{Referências}

Barbosa, G. R. (2020). Disponibilidade de estudantes de oito cursos da saúde de uma universidade pública brasileira para a educação interprofissional. (88 p.) Tese (doutorado) - Universidade Estadual de Campinas, Faculdade de Ciências Médicas.

Correa, C. P. S., Hermuche, L. S., Luccheti, A. L. G., Ezequiel, O. S., \& Luccheti, G. (2019). Current status of brazilian interprofessional education: a national survey comparing physical therapy and medical schools. Rev Assoc Med Bras, 65(10):1241-8.

Dias, H. S. (2013). A trajetória da política nacional de reorientação da formação profissional em saúde no SUS. Cien Saude Colet., 18(6):1613-24.

Dyess, A. L., Brown, J. S., Brown, N. D., Flautt, K. M., \& Barnes, L. J. (2019). Impact of interprofessional education on students of the health professions: a systematic review. J Educ Eval Health Prof, 16:1-6.

Facchini, L A., Tomasi, E., \& Dilélio, A. S. (2018). Qualidade da atenção primária à saúde no Brasil: avanços, desafios e perspectivas. Saúde em Debate, 42(1):208-23. 
Research, Society and Development, v. 10, n. 9, e10910917997, 2021

(CC BY 4.0) | ISSN 2525-3409 | DOI: http://dx.doi.org/10.33448/rsd-v10i9.17997

Furr, S. (2020). Understanding roles in health care through interprofessional education experiences. British Journal of Nursing, 29(6):364-72.

Kanji, Z., Lin, D., \& Karan, J. (2020). Assessing dental hygiene students' readiness for interprofessional learning and collaborative practice. J Dent Educ, 84(6):669-80.

Lakatos, E. V., \& Marconi, M. A. (2010). Metodologia científica. (7a ed.), Atlas.

Lima, A. W. S., Alves, F. A. P., Linhares, F. M. P., Costa, M. V., Coriolano-Marinus, M. W. L., \& Lima, L. S. (2020). Perception and manifestation of collaborative competencies among undergraduate health students. Rev Lat Am Enfermagem, 28.

Ministério da Saúde, Brasil. (2012). Departamento de Atenção Básica. Política Nacional de Atenção Básica. Atlas.

Ministério da Saúde, Brasil. (2018). Programa de Educação pelo Trabalho para a Saúde: PET-Saúde Interprofissionalidade. Atlas.

Nguyen, J., Smith, L., Hunter, J., \& Harnett, J. (2019). Conventional and complementary medicine health care practitioners' perspectives on interprofessional communication: A qualitative rapid review. Medicina, 55(10):1-17.

Parsell, G., \& Bligh, J. (1999). The development of a questionnaire to assess the readiness of health care students for interprofessional learning (RIPLS). Medical Education, 33(2):95-100.

Peduzzi, M., Norman, I. J., Germani, A. C. C. G., Silva, J. A. M., \& Souza, G. C. (2013). Interprofessional education: training for healthcare professionals for teamwork focusing on users. Rev da Esc Enferm, 47(4):977-83.

Peduzzi, M., Norman, I. J., Coster, S., \& Meireles, E. (2015). Cross-cultural adaptation of the Readiness for Interprofessional Learning Scale in Brazil. Rev da Esc Enferm, 49(2):7-14.

Queiroz, R. S., \& Valente, G. S. C. (2019). Diagnóstico situacional em unidade básica de saúde: contribuições para o campo da saúde coletiva. Rev Enferm Atual in Derme, 88(26).

Ribeiro, L. C. C., Ribeiro, M., Dias, K. S., Matos, K. A., \& Ferreira, T. S. (2008). O diagnóstico administrativo e situacional como instrumento para o planejamento de ações na estratégia saúde da família. Cogitare Enferm, 13(3):448-52.

Ribeiro, P. C., Pedrosa, J. I. S., Nogueira, L. T., \& Souza M. F. (2012). Ferramentas para o diagnóstico comunitário de saúde na consolidação da estratégia saúde da família. Revista Tempus Actas de Saúde Coletiva, 6(4):161-74.

Reeves, S., Perrier, L., Goldman, J., Freeth, D., \& Zwarenstein, M. (2013). Interprofessional education: effects on professional practice and healthcare outcomes. Cochrane Database of Systematic Reviews.

Sagahutu, J. B., Kagwiza, J., Cilliers, F., \& Jelsma, J. (2020). The impact of a training programme incorporating the conceptual framework of the International Classification of Functioning (ICF) on behaviour regarding interprofessional practice in Rwandan health professionals: A cluster randomized control trial. PLoS One, 15(2):1-17.

Silva, C. S. S. L., Koopmans, F. F., \& Daher, D. V. (2016). O diagnóstico situacional como ferramenta para o planejamento de ações na atenção primária a saúde. Rev Pró-UniverSUS, 7(2):30-3.

Skolka, M., Hennrikus, W. L., Khalid, M., \& Hennrikus, E. F. (2020). Attitude adjustments after global health inter-professional student team experiences. Medicine, 99(16):1-5.

Vasconcelos, N. B. (2010). Programa nacional de assistência estudantil: uma análise da evolução da assistência estudantil ao longo da história da educação superior no Brasil. Ensino em Revista, 17(2):599-616.

Wang, Z., Feng, F., Gao, S., \& Yang, J. (2019). A systematic meta-analysis of the effect of interprofessional education on health professions students' attitudes. J Dent Educ, 83(12):1361-9.

World Health Organization. (2010). Framework for action on interprofessional education \& collaborative practice. WHO. 